\title{
A Project to Improve Management of Biomedical Equipment in Selected Units of District General Hospital, Gampaha,
} Sri Lanka

\author{
PD Koggalage ${ }^{1}$, Sunil De Alwis ${ }^{2}$
}

\begin{abstract}
Introduction: It is vital to manage biomedical equipment efficiently in planning, acquisition, maintenance, repair, and disposal stages. It was observed and experienced that there were issues in management of biomedical equipment in District General Hospital (DGH), Gampaha, which is a provincial tertiary care hospital in Sri Lanka.

Aim: To identify the gaps and improve the existing system for management of biomedical equipment in the Operation Theaters (OTs) A and B, Medical Intensive Care Unit (MICU), and Ward No. 1 of DGH, Gampaha.

Materials and methods: This interventional research project was conducted in three phases in three randomly selected units: MICU, OTs A and B, and Ward No. 1. The preinterventional phase identified the gaps in the existing system through key informant interviews, desk review of documents, and surveys. Based on the findings, interventions were designed and implemented in the second phase. The postinterventional phase assessed the effectiveness of the interventions using the same techniques in phase I.

Results: Lack of a systematic mechanism to record and quickly access information required for equipment management and incompleteness of information was found. A central-level computer-based Biomedical Equipment Inventory Management System (BEIMS) was implemented along with a personal record keeping system at the unit level. The postinterventional evaluation revealed that the interventions made the vital information available and significantly improved quick accessibility to necessary information about biomedical equipment $(p<0.05)$. Also, there was a significant improvement in the level of convenience $(p<0.05)$ and satisfaction $(p<0.05)$ of the stakeholders with the new mechanism. Conclusion: The BEIMS was effective in improving the management of biomedical equipment. It is recommended to link the BEIMS online with the respective units to improve accessibility to information and also to replicate this project in other units of the hospital.

Keywords: Biomedical equipment, Biomedical equipment inventory management, Health technology management, Inventory management system, Medical equipment management.

International Journal of Research Foundation of Hospital and Healthcare Administration (2019): 10.5005/jp-journals-10035-1109
\end{abstract}

\section{INTRODUCTION}

Health technologies are of utmost importance in day-to-day functioning of health system. Nowadays, with the continuous advancement in health technologies, different types of medical devices/equipment are being used in the healthcare setup in the prevention, diagnosis, and treatment of illness and disease as well as patient rehabilitation. Demand for medical equipment is increasing day by day. At the same time, costs are also increasing. ${ }^{1}$

\section{Health Technology}

It is the application of organized knowledge and skills in the form of devices, medicines, vaccines, procedures, and systems developed to solve a health problem and improve quality of life. ${ }^{2}$

\section{Medical Device}

An article, instrument, apparatus, or machine that is used in the prevention, diagnosis, or treatment of illness or disease, or for detecting, measuring, restoring, correcting, or modifying the structure or function of the body for some health purpose. ${ }^{2}$ Medical devices range from small, inexpensive items such as thermometers to expensive complex items such as computerized tomography (CT) scanners, magnetic resonance image (MRI) scanners, and radiosurgery equipment costing several millions of dollars. ${ }^{3}$

\footnotetext{
1,2Ministry of Health and Indigenous Medical Services, Colombo, Western Province, Sri Lanka

Corresponding Author: PD Koggalage, Ministry of Health and Indigenous Medical Services, Colombo, Western Province, Sri Lanka, Phone: +94718766870, e-mail: dineshkoggalage@yahoo.com

How to cite this article: Koggalage PD, De Alwis S. A Project to Improve Management of Biomedical Equipment in Selected Units of District General Hospital, Gampaha, Sri Lanka. Int J Res Foundation Hosp Healthc Adm 2019;7(2):67-74.

Source of support: Nil

Conflict of interest: None
}

\section{Medical equipment}

These are interchangeably known as "biomedical equipment". They are the medical devices that require calibration, maintenance, repair, user training, and decommissioning which are usually managed by biomedical engineers. Implantable, disposable, or single-use medical devices are excluded from medical equipment. $^{2}$

Healthcare providers should provide an efficient health service of high quality and safety, and the services should be economical as

() The Author(s). 2019 Open Access This article is distributed under the terms of the Creative Commons Attribution 4.0 International License (https://creativecommons. org/licenses/by-nc/4.0/), which permits unrestricted use, distribution, and non-commercial reproduction in any medium, provided you give appropriate credit to the original author(s) and the source, provide a link to the Creative Commons license, and indicate if changes were made. The Creative Commons Public Domain Dedication waiver (http://creativecommons.org/publicdomain/zero/1.0/) applies to the data made available in this article, unless otherwise stated. 
well. Having the appropriate medical equipment in the right place at the right time, fit for purpose, and well maintained is essential for the delivery of good quality healthcare. Hence, it is vital that the healthcare providers pay more attention on efficient and appropriate utilization of biomedical equipment. ${ }^{3}$

\section{Management of Biomedical Equipment}

Majority of medical equipment are technically complex and require specific expertise to use, maintain, and repair. The unavailability or failure of medical equipment can lead to significant risks to patients and the staff as well. Therefore, it is the responsibility of any healthcare provider to manage their own medical equipment which includes planning, acquisition, maintenance, repair, and disposal. ${ }^{3}$

\section{Biomedical Equipment Inventory}

The inventory is a working document that is regularly checked and updated to accurately reflect the status of healthcare technology assets. ${ }^{2}$ The first step in managing biomedical equipment is to develop an inventory. When the inventories are regularly updated and maintained, they serve as an important and powerful tool to improve management of key aspects of biomedical equipment. ${ }^{2}$

\section{District General Hospital, Gampaha}

It is the largest provincial hospital in the Western province of Sri Lanka, and it provides outpatient and inpatient services. Outpatient Department and the Preliminary Care Unit play a significant role in providing outpatient services. The hospital has 19 wards providing curative services under major clinical specialties and under some subspecialties. The MICU, Surgical Intensive Care Unit, and the Coronary Care Unit serve the critically ill while the needy newborns are cared at the Neonatal Unit. The hospital has five OTs. All these units are equipped with a large number of biomedical equipment.

\section{Statement of the Problem}

Biomedical equipment are important assets in diagnosing, treating, monitoring, and rehabilitating patients with various diseases. Most vital decisions on management of patients are heavily dependent on biomedical equipment. DGH, Gampaha, as a tertiary care provider in Sri Lanka, with numerous high-tech biomedical equipment was facing many issues in day-to-day management of them. Lack of proper mechanisms at the central as well as at the unit level to record and quickly access necessary information, which are important at every stage of the equipment management cycle was the main highlighted problem.

There was no proper systematic mechanism to maintain records and quickly access vital information required for efficient management of biomedical equipment at DGH, Gampaha.

\section{Justification}

Similar to other countries, in Sri Lanka also, the demand for biomedical equipment has been increasing day by day with the advancement in medical technologies. At the same time, their costs have also been rising, and the gap between needs and resources have been widening. Hence, it is important to use and maintain the available biomedical equipment in a cost-effective manner to maximize their utilization. On the other hand, suboptimal usage and maintenance of them may lead to wrong outputs and also challenge safety of the patient and the user. ${ }^{4}$

The DGH, Gampaha, is also equipped with many hightech biomedical equipment which are of high cost. However, it had been observed and experienced that there were issues in efficient management of them at various stages of their life cycle as there was no proper mechanism to record and quickly access information needed for the management of biomedical equipment. The World Health Organization (WHO) emphasized that the first step in managing biomedical equipment is to develop an effective record keeping mechanism which will serve as a powerful tool to improve the management of key aspects of biomedical equipment. ${ }^{2}$

Therefore, it was a very important and a timely requirement to conduct this research project in order to identify the gaps in the system for management of biomedical equipment and also to design and implement suitable interventions to improve the management of biomedical equipment in DGH, Gampaha.

\section{Objectives}

\section{General Objective}

To improve the existing system for management of biomedical equipment in the OTs A and B, MICU, and Ward No. 1 of DGH, Gampaha, Sri Lanka

\section{Specific Objectives}

- To describe the current processes, practices, and perceptions of the health staff to identify gaps in the existing system for management of biomedical equipment in OTs A and B, MICU, and Ward No. 1 of DGH, Gampaha;

- To design and implement interventions to address the identified gaps in order to improve the existing system for the management of biomedical equipment in OTs A and B, MICU, and Ward No. 1 of DGH, Gampaha;

- To evaluate the effectiveness of the interventions to improve the existing system for the management of biomedical equipment in OTs A and B, MICU, and Ward No. 1 of DGH, Gampaha.

\section{Materials and Methods}

\section{Project Design}

This interventional research project was phased out as follows.

Phase I: In this phase, the existing processes and practices were reviewed and analyzed and also gaps and issues were identified in the management of biomedical equipment.

Phase II: During this phase, interventions were developed and implemented to address the identified gaps and issues.

Phase III: Effectiveness of implemented interventions to address the gaps and issues was evaluated to measure the success of the project.

\section{Project Setting}

This project was conducted in DGH, Gampaha, in the three randomly selected units: MICU, Ward No. 1, and OTs A and B.

\section{Project Duration}

This project was conducted over a period of 15 months starting from April 2018 to June 2019.

\section{Project Stakeholders}

The director, deputy director, two medical officers (quality management and planning), surgical pharmacist in-charge of biomedical equipment (BME), management assistant in-charge of BME, four consultants in selected units, four in-charge nursing 
officers in selected units, four special grade nursing officers, and eighteen nursing officers in selected units involving in the management of BME were selected as stakeholders (a total of 35) of the project, depending on their involvement at different stages of the management cycle of biomedical equipment. No sampling method was applied.

\section{Project Instruments/Techniques}

During phases I and III, following quantitative and qualitative research methods were applied:

- Key informant interviews (KIls) with the director/DGH, Gampaha, the surgical pharmacist handling management of biomedical equipment and a nursing sister in-charge of three selected units using "interviewer guides".

- Desk review of documents by perusing biomedical equipment inventories and records related to the management of biomedical equipment.

- Surveys using a structured self-administered questionnaire with selected group of project stakeholders as mentioned above. It included 35 participants from different staff categories.

- Biomedical equipment survey using a "checklist format" to identify the availability of selected details about biomedical equipment in selected three units.

The interviewer guides, checklist formats, and the questionnaires were developed following discussions with experts in the field and review of literature.

\section{Collection of Data and Analysis}

Data collection was done by the principal investigator. Survey data were analyzed using Statistical Package for Social Sciences (SPSS) software. Paired $t$ test was the statistical test applied to check the statistical significance of the pre- and postinterventional parameters. Means for the parameters were calculated by assigning a score to each response depending on the favorability with the statement (more favorable means higher score and less favorable means lower score).

For example,

\begin{tabular}{ll}
\hline - Very convenient/very satisfied & 05 points \\
- Convenient/satisfied & 04 points \\
- Somewhat convenient/somewhat satisfied & 03 points \\
- Inconvenient/dissatisfied & 02 points \\
- Very inconvenient/very dissatisfied & 01 points \\
\hline
\end{tabular}

Key informant interviews were recorded with the consent for easy compilation, and narrative analysis was done.

\section{Project Management}

Project Planning

The information gathered during phase I was used to identify the problem to be addressed by the project. The best possible solutions were identified and a package of interventions to improve the existing system for management of biomedical equipment was developed accordingly (refer to the section on Results). The following activities were carried out:

- Identification of resources to develop planned interventions [e.g., information technology (IT) support] after discussing with director and Medical Officer (Planning).
- Feasibility assessment of proposed interventions was conducted through discussions with relevant stakeholders.

- Biomedical equipment survey was conducted to collect relevant details of the equipment in the selected units using a "checklist" format which also gathered information about the availability of relevant details about equipment. The checklist was developed using the WHO inventory data collection form as a guiding document. $^{2}$

- Selection of equipment to be included in the inventory was carried out based on the inclusion criteria developed using Fennigkoh and Smith Model for inventory inclusion of biomedical equipment. This was customized to the local setting with inclusion of the approximate value of the equipment at acquisition after discussing with relevant stakeholders of the project (the director and the surgical pharmacist).

\section{Project Execution}

Following strategies were used to implement this project.

- Establishment of a focal point-The surgical pharmacist who has been handling the biomedical equipment was identified as the focal point for this project.

- Assign responsibility at unit level-The responsibility and coordination at unit levels were assigned to the in-charge nursing sister of the respective unit.

- Obtaining IT support-The necessary IT support to implement the interventions was obtained from an expert in the field.

- Training of staff-An orientation training was conducted for the surgical pharmacist in-charge and in-charge nursing sisters in three selected units about the usage of interventions.

\section{Project Evaluation}

The effectiveness of the project was evaluated during phase III using the same techniques in phase I.

\section{Administrative and Ethical Requirements}

Approval for the project proposal was obtained from the Board of Study in Medical Administration of the Postgraduate Institute of Medicine (PGIM), University of Colombo. Permission was obtained from the Regional Director of Health Services, Gampaha and Director of DGH, Gampaha was taken.

Ethics approval was obtained from the Ethics Review Committee, PGIM. Informed consent was taken from all participants using an information sheet. Confidentiality of information and anonymity of participants were preserved.

\section{Results}

The qualitative and quantitative techniques used in this research project revealed the following findings. They are described under the three phases of the project.

\section{Phase I-Preinterventional Assessment}

The preinterventional KIls conducted with the director, surgical pharmacist in-charge of biomedical equipment and the nursing sister of the selected unit identified the existing processes and practices involved in the management of biomedical equipment and issues/gaps in the processes. 


\section{Identification of Gaps}

The preinterventional KII revealed that there was no proper systematic mechanism to record and access information required for management of biomedical equipment in the hospital.

The director mentioned that timely availability of accurate information is vital in every stage of equipment management cycle. He said that the existing record keeping mechanism has some inefficiencies such as lack of vital information and difficulty in quickly accessing information about location, condition/level of functioning, services, maintenance details, etc., of an equipment. Therefore, he emphasized the importance of easily and quickly retrievable information system to facilitate optimum management of biomedical equipment.

The surgical pharmacist and the nursing sister mentioned that there was no proper system to maintain separate records for each equipment at unit/ward level. Further, the nursing sister mentioned that the lack of relevant information at unit level led to inefficiencies in management of biomedical equipment. Personal records and tagging systems were not available for each and every equipment. The surgical pharmacist reiterated that the existing record keeping mechanism was incomplete and completely paper based, hence had difficulties in accessing required information quickly. Also, sorting, filtering, and searching relevant information was difficult with the manual system.

Therefore, the following could be identified as the main gaps in the process of managing biomedical equipment.

- Lack of a systematic mechanism to easily and quickly access information (e.g., location, functionality, details about service/maintenance) about biomedical equipment at the central level.

- Lack of important details (data completeness) in the existing system (e.g., condition/level of functioning, service-related details, etc.)

- Lack of a systematic mechanism to maintain separate "record files" for each equipment at the unit/ward level.

- Unavailability of "identification tags" for each selected equipment.

\section{Biomedical Equipment Survey}

As mentioned earlier, a biomedical equipment survey was conducted in selected three units using the checklist format.

All checklist and survey results in phase I are described under postinterventional section (phase III) for easy comparison.

\section{Phase II-Design and Implementation of Interventions}

A package of interventions was developed and implemented to address the gaps identified in phase I after several rounds of discussions with the director, surgical pharmacist, and the selected nursing sister.

- Intervention 1-A central-level computer-based BEIMS was developed using Microsoft Access software as shown in Figures 1 and 2.

The system included the following key features.

- A data entry interface with general information, service, maintenance details, and availability details of biomedical equipment

- A facility to generate a summary report of each equipment entered into the inventory

- A facility to search equipment quickly with ID or serial number

- Facilities to sort equipment according to different parameters (e.g., location, functionality, etc.)

Selection of equipment to be included in the BEIMS was done in biomedical equipment survey. Accordingly, a total of 55 equipment were identified from three selected units as follows.

- OTs $A$ and $B-28$

- MICU-15

- Ward 01-12

- Intervention 2-A "record file" was introduced for each selected equipment included in the BEIMS. This record file was maintained at the respective units by the in-charge officers and consisted of information sheet generated by the BEIMS with all

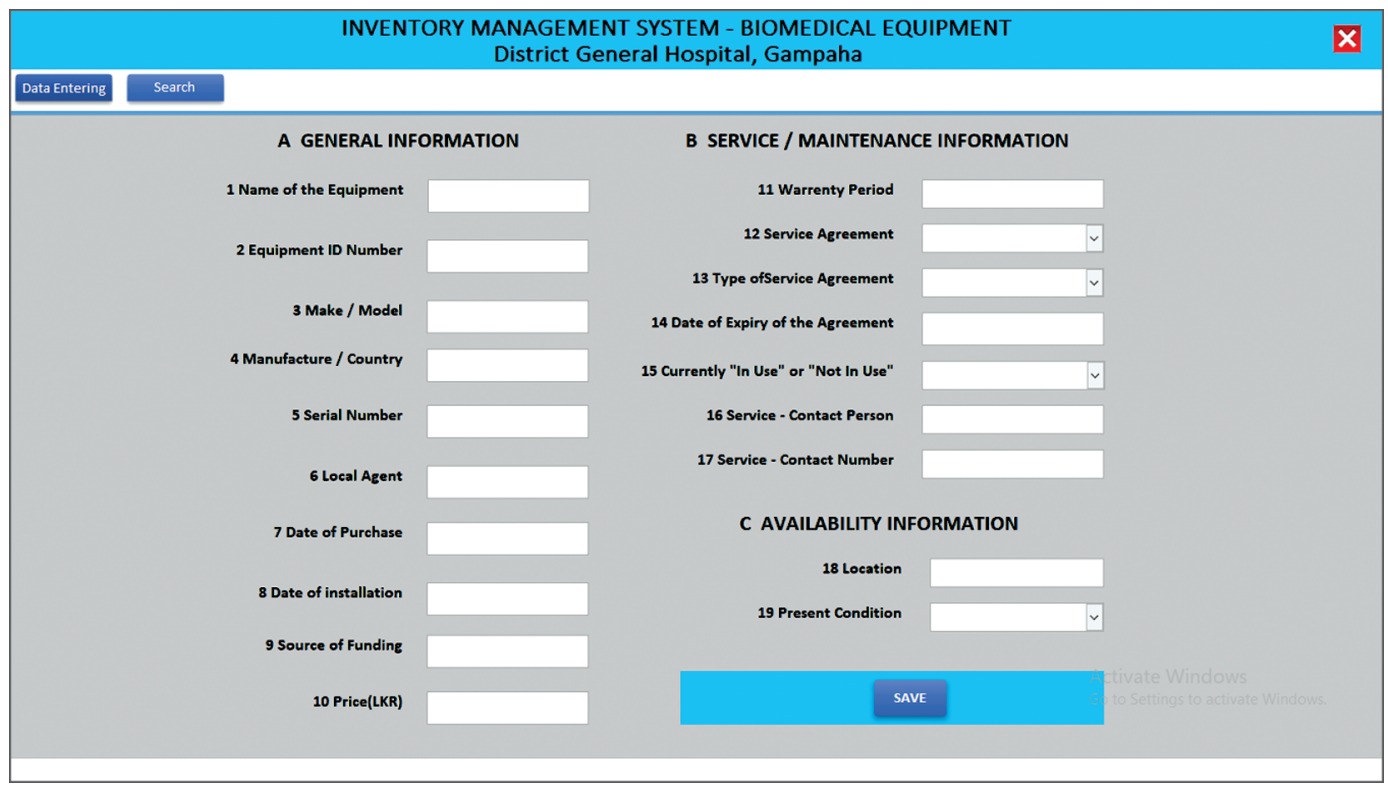

Fig. 1: Biomedical Equipment Inventory Management System—Data entry interface 


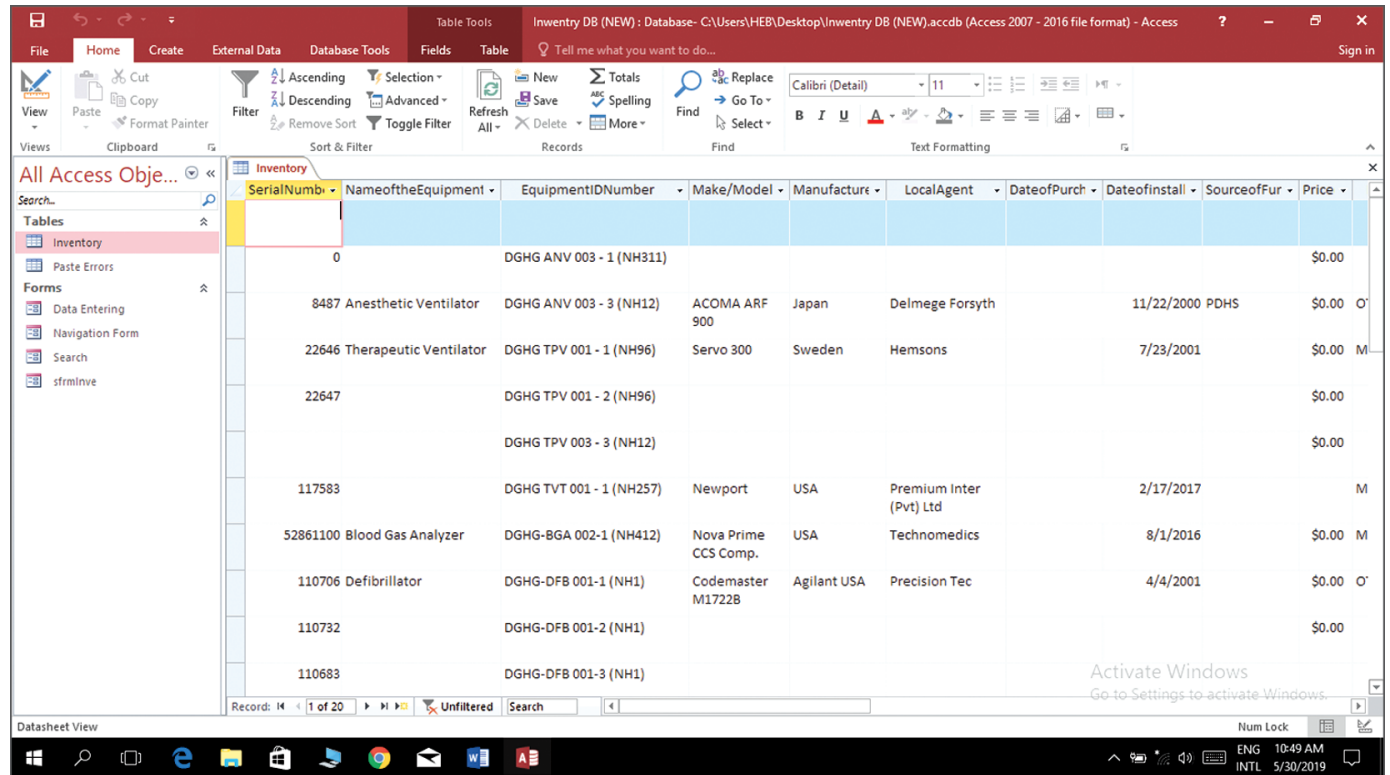

Fig. 2: Biomedical Equipment Inventory Management System—Database

Table 1: Frequency distribution of selected biomedical equipment according to the availability of selected key details/ features before and after the interventions $(n=55)$

\begin{tabular}{|c|c|c|c|c|}
\hline \multirow[b]{2}{*}{ Details/features } & \multicolumn{2}{|c|}{ Preinterventional } & \multicolumn{2}{|c|}{ Postinterventional } \\
\hline & Yes (\%) & No (\%) & Yes (\%) & No (\%) \\
\hline Personal record file at unit level & $12(21.8)$ & $43(78.8)$ & $55(100.0)$ & $00(0.0)$ \\
\hline Identification tag & $08(14.5)$ & $47(85.5)$ & $55(100.0)$ & $00(0.0)$ \\
\hline Warranty details & $15(27.3)$ & $40(72.7)$ & $55(100.0)$ & $00(0.0)$ \\
\hline Service agreement details & $12(21.8)$ & $43(78.8)$ & $50(90.9)$ & $05(9.1)$ \\
\hline Type of service agreement & $12(21.8)$ & $43(78.8)$ & $50(90.9)$ & $05(9.1)$ \\
\hline Service contact person's name & $08(14.5)$ & $47(85.5)$ & $55(100.0)$ & $00(0.0)$ \\
\hline Service contact telephone number & $08(14.5)$ & $47(85.5)$ & $55(100.0)$ & $00(0.0)$ \\
\hline Present location & $53(96.4)$ & $02(3.6)$ & $55(100.0)$ & $00(0.0)$ \\
\hline Present condition & $12(21.8)$ & $43(78.8)$ & $55(100.0)$ & $00(0.0)$ \\
\hline
\end{tabular}

relevant details of the equipment including a record of services and maintenance and any other relevant documents.

- Intervention 3-An "identification tag" was introduced for each selected equipment for easy access to relevant details.

The users were given an orientation training on how to use and maintain the new mechanisms.

\section{Phase III-Postinterventional Assessment}

Effectiveness of the interventions to address the identified gaps was assessed and the results are shown below.

\section{Findings of Klls}

In comparison to phase I, the postinterventional KIls with the same participants revealed the following. The director mentioned that the newly established computer-based BEIMS had made the centrallevel inventory management more systematic. He further said that the required information are readily accessible with the new system.

The surgical pharmacist mentioned that the data entry interface of the system was very user-friendly and contained vital information about general identification, service/maintenance, and availability of the equipment which are important for efficient management. Also, the surgical pharmacist stated that the editing, sorting, searching, and printing facilities in the system were very useful and convenient for recording and quick accessibility to information. The record sheet generated by the system with key information for each equipment was helpful to provide information to be included in personal records at the unit level. However, the surgical pharmacist emphasized that the system can be updated with all required information with the receipt of new equipment as most of the old equipment lack some information.

The nursing sister also, in agreement with other key informants said that the new system was very useful for maintaining records for biomedical equipment. The unit-level personal record system and the identification tags had helped them to maintain systematic records for each selected equipment. Nevertheless, the nursing sister mentioned that if the central-level computer-based system can be extended to unit/ward level, it would be more efficient in biomedical equipment management in the whole hospital.

\section{Checklist and Survey Results}

Of the 35 stakeholders considered, 32 (91.4\%) and 29 (82.9\%) responded in pre- and postinterventional surveys, respectively.

Availability of selected details/features in the mechanisms before and after the interventions for the management of biomedical equipment: Table 1 shows the results of checklist data on the availability of selected key details/features in records for each 
Table 2: Significance test statistics for differences in responses of participants in pre- and postinterventional assessments on timeliness of selected processes

\begin{tabular}{llll}
\hline & \multicolumn{1}{c}{ Mean (SD) } & \multicolumn{1}{c}{ Significance $(p$ value $)$} \\
\cline { 2 - 3 } Indicator & Preinterventional $(n=32)$ & Postinterventional $(n=29)$ & 0.000 \\
\hline $\begin{array}{l}\text { Average time taken to obtain information required for } \\
\text { management of equipment }\end{array}$ & $4.93(0.47)$ & $3.86(0.36)$ & 0.090 \\
$\begin{array}{l}\text { Timeliness of services/maintenance of biomedical } \\
\text { equipment in hospital/unit }\end{array}$ & $2.89(0.67)$ & $3.14(0.44)$ & 0.000 \\
$\begin{array}{l}\text { Frequency of delays in service and maintenance due to } \\
\text { nonavailability of information }\end{array}$ & $3.55(0.51)$ & $2.28(0.45)$ & \\
\hline
\end{tabular}

Table 3: Significance test statistics for differences in responses of participants on level of convenience and usefulness of pre and postinterventional mechanisms to access information

\begin{tabular}{llll}
\hline & \multicolumn{2}{c}{ Mean (SD) } \\
\cline { 2 - 3 } Indicator & Preinterventional $(n=32)$ & Postinterventional $(n=29)$ & Significance $(p$ value $)$ \\
\hline $\begin{array}{l}\text { Convenience to obtain information about a particular } \\
\text { equipment }\end{array}$ & $2.41(0.73)$ & $4.37(0.68)$ & 0.000 \\
$\begin{array}{l}\text { User-friendliness of the mechanism to staff in managing } \\
\text { biomedical equipment in the selected units }\end{array}$ & $2.34(0.67)$ & $4.00(0.71)$ & 0.000 \\
$\begin{array}{l}\text { Usefulness of the mechanism to facilitate management } \\
\text { of biomedical equipment in selected units }\end{array}$ & $2.21(0.67)$ & $4.10(0.67)$ & 0.000
\end{tabular}

Table 4: Significance test statistics for differences in responses on the level of satisfaction about the pre- and postinterventional mechanisms

\begin{tabular}{llll}
\hline & \multicolumn{2}{c}{ Mean $(S D)$} \\
\cline { 2 - 3 } Indicator & Preinterventional $(n=32)$ & Postinterventional $(=29)$ & Significance $(p$ value $)$ \\
\hline Satisfaction on quick availability of relevant information & $2.72(0.75)$ & $4.10(0.67)$ & 0.000 \\
required for equipment management & & & 0.000 \\
Satisfaction on convenience of the mechanism & $2.66(0.61)$ & $4.17(0.71)$ & 0.000 \\
Overall satisfaction about the mechanisms & $2.89(0.90)$ & $4.37(0.68)$ & 0 \\
\hline
\end{tabular}

selected biomedical equipment before and after the intervention. Accordingly, personal records (100\%) and identification tags (100\%) were made available for each selected equipment after the intervention. Other key details such as service agreement details (90.9\%), type of service agreement (90.9\%), service person's contact details (100\%), present location (100\%), and present condition (100\%) which were lacking before the intervention were made available after implementing the BEIMS.

Timeliness of selected processes in biomedical equipment management:Table 2 presents significance test statistics of pre- and postinterventional perceptions of participants on the timeliness of the selected processes. A significant difference $(p<0.05)$ was observed in the reduction of the average time consumed to obtain information and the frequency of delays in services/maintenance due to nonavailability of information. However, the improvement in timeliness of services/maintenance was not statistically significant $(p>0.05)$.

Level of convenience and usefulness of the mechanism: The significance test statistics on responses of participants on levels of convenience and usefulness of the pre- and postinterventional mechanisms are depicted in Table 3. A significant improvement $(p<0.05)$ was observed in levels of convenience, user-friendliness, and usefulness of the pre- and postinterventional mechanisms to access information about biomedical equipment.

Level of satisfaction about the mechanism: The significance test statistics for the levels of satisfaction of participants about the pre- and postinterventional mechanisms are presented in Table 4. A significant increase $(p<0.05)$ was observed in the levels of satisfaction on quick accessibility to required information and the convenience of the mechanism. Also, the increase in their overall satisfaction was statistically significant $(p<0.05)$.

\section{Discussion}

With the rapid advancement in health technologies, different types of biomedical equipment are being used in healthcare setup in the prevention, diagnosis, and treatment of illness and disease as well as patient rehabilitation. Demand and the costs for medical equipment are increasing day by day. ${ }^{1}$ Therefore, it is very important to manage biomedical equipment effectively for maximum utilization. Furthermore, proper management is important in order to render best quality and safe healthcare for patients while maintaining equipment in the best cost-effective manner. ${ }^{5}$

Inventory management and documentation is one of the important assistive stages in the life cycle. ${ }^{6}$ This can be considered as the first step in designing an effective equipment management program in any organization. Hence, each healthcare organization should maintain an inventory for medical equipment. ${ }^{7}$ The WHO also has identified medical equipment inventory as an essential part of an effective healthcare technology management system. ${ }^{2}$ However, lack of a systematic mechanism at the central as well as the unit level to easily access information and incompleteness of available information was identified as the key issue in the management of biomedical equipment in DGH, Gampaha. 
The existing mechanism was completely paper based and lacking some vital information. This had resulted in some inconveniences and inefficiencies in managing biomedical equipment in the selected units of the hospital. A similar research project conducted in primary and secondary healthcare institutions in the regional directorate of health services (RDHS), Gampaha, Sri Lanka, to improve the maintenance of biomedical equipment found lack of medical equipment inventory, guiding principles for decisionmaking, proper information, communication and record keeping methods and capacity building and training for the staff as main reasons for poor maintenance program. ${ }^{8}$

In the present research project, the central-level BEIMS was developed using MS Access with key information. It had the ability to quickly access information of a particular equipment. Also, it had facilities for searching, sorting, filtering, and printing information sheets. Furthermore, a separate record keeping mechanism and tagging system was introduced at the unit level to supplement the central inventory. The whole system comprised general, service/ maintenance-related and availability-related information about selected equipment. The previous research project conducted in RDHS, Gampaha, also developed a medical equipment inventory, a guideline including prioritization, information, communication, and record keeping criteria for medical equipment management program as interventions. ${ }^{8}$

The postinterventional KIIs revealed that the BEIMS at the central level and the record keeping mechanism at the unit level had made the management of biomedical equipment more convenient and efficient. Further, the system was user-friendly and contained essential information required by the staff to facilitate management of equipment. With the searching, sorting, filtering, and printing facilities, the computer-based system had made access to information more efficient. Similar to this, the previous project in RDHS, Gampaha also found significant improvements in maintenance activities carried out by primary and secondary care institutions. $^{8}$

With the establishment of the BEIMS, many of the important information of equipment like general identification details, locations, and service/maintenance details were made available for selected equipment. The medical equipment management guideline of the MedPro Group also states to include unique identification number, the equipment manufacturer, model number and serial number, description of the equipment, location of the equipment (for equipment generally kept in a fixed location), identity of the department considered to "own" the equipment in the inventory. ${ }^{4}$

According to the perception of the stakeholders of the present research project, the average time taken to obtain necessary information about an equipment had significantly reduced with the implementation of interventions $(p=0.000)$ (Table 2). Also, they perceived that the frequency of delays in services/ maintenance due to unavailability information had significantly reduced ( $p=0.000$ ) (Table 2 ). The WHO has also mentioned that when inventories are regularly updated and maintained, they improve the management of these aspects of biomedical equipment. Even though, the perceived timeliness of services/ maintenance had improved, it was not statistically significant ( $p=$ 0.090) (Table 2). This may be due to many other factors that affect timeliness of servicing/maintenance of equipment such as delays in procurement processes and approval processes, etc. which should be further researched. The parameters considered were measured by staff perceptions as an alternative proxy measure due to the limited time.

The convenience to access information and the user-friendliness had increased after implementation of the new system and the increase was statistically significant $(p=0.000$ ) (Table 3). The new system was more useful to facilitate the management of biomedical equipment compared to the manual paper-based system ( $p=$ 0.000) (Table 3). The project conducted in RDHS, Gampaha, also found a significant improvement in the perception of the staff regarding the above aspects in the management of biomedical equipment. ${ }^{8}$ The staff satisfaction on convenience and quick accessibility to information and also their overall satisfaction about the mechanism had significantly improved with the new system ( $p=0.000)$ (Table 4).

With the above findings, it was evident that the newly established mechanisms have improved the system for management of biomedical equipment in selected units of the hospital. However, an assessment of a longer duration is required for more detailed evaluation of effectiveness.

\section{Limitations}

The research project was confined to selected units of DGH, Gampaha, due to feasibility issues. Also, only the selected equipment were included in the BEIMS due to limited time duration of the project.

The effectiveness of the project was assessed mainly through proxy indicators using the questionnaire as a longer time period is required to assess the real outcome of the intervention.

The linking of the BEIMS with the respective units was unable due to technical limitations.

\section{Conclusion and Recommendations}

\section{Conclusion}

Lack of a systematic mechanism to quickly access necessary information and unavailability of vital information required for management of biomedical equipment were identified as key issues in DGH, Gampaha.

Considering the above gaps a computer-based central BEIMS, the unit-level record file system and identification tag system for biomedical equipment were designed and implemented in the MICU, Ward No. 1, and OTs A and B.

The interventions were effective in improving quick accessibility to necessary accurate information about a particular biomedical equipment and, in turn, helped management of biomedical equipment. Also, a significant improvement was observed in the level of convenience and satisfaction of the stakeholders about the newly established mechanism to improve the management of biomedical equipment.

\section{Recommendations}

It is recommended to link the BEIMS online with the respective units to further improve accessibility to information. The mechanism should be further improved by incorporating comprehensive service/maintenance schedules for each biomedical equipment, with an alert system to users as this will improve timely servicing of equipment.

It is desirable to implement this project in other units of the hospital as well. So that the management of biomedical equipment in the whole hospital can be improved. 


\section{References}

1. Ministry of Health Republic of Zambia. Medical Equipment Management Guidelines. Medical Equipment Management Guidelines. Republic of Zambia: Ministry of Health, Republic of Zambia, September 2012.

2. World Health Organization. Introduction to medical equipment inventory management: WHO Press, World Health Organization, 20 Avenue Appia, 1211 Geneva 27, Switzerland, 2011.

3. Office of the Auditor General.Management of Medical Equipment. Western Australia: Office of the Auditor General, 2017.

4. MedPro Group.Guideline: Medical Equipment Management. Fort Wayne: MedPro Group, 2016.
5. Aruna M, Uma G, Saadia N. Efficient equipment management for biomedical engineering. Int Rev Manage Market 2018;8(3): 69-74.

6. Saleh N. Medical Equipment Management. Asian Hospital \& Healthcare Management. [Online] 2019. https://www.asianhhm.com/ technology-equipment/medical-equipment-management.

7. MedPro Group. Medical Equipment Management. Fort Wayne: MedPro Group, 2019.

8. Perera A, A project to assess and improve the medical equipment maintenance program of Gampaha regional director of health service division [dissertation]. Postgraduate Institute of Medicine, University of Colombo; 2018 